\title{
Dimensionality dependence of the wave-function statistics at the Anderson transition
}

\author{
A. Mildenberger, ${ }^{1,2}$ F. Evers, ${ }^{2}$ and A. D. Mirlin ${ }^{1,2, *}$ \\ ${ }^{1}$ Institut für Theorie der Kondensierten Materie, Universität Karlsruhe, 76128 Karlsruhe, Germany \\ ${ }^{2}$ Institut für Nanotechnologie, Forschungszentrum Karlsruhe, 76021 Karlsruhe, Germany
}

(Received 3 May 2002; published 29 July 2002)

\begin{abstract}
The statistics of critical wave functions at the Anderson transition in three and four dimensions are studied numerically. The distribution of the inverse participation ratios (IPRs) $P_{q}$ is shown to acquire a scale-invariant form in the limit of large system size. Multifractality spectra governing the scaling of the ensemble-averaged IPRs are determined. Conjectures concerning the IPR statistics and the multifractality at the Anderson transition in a high spatial dimensionality are formulated.
\end{abstract}

DOI: 10.1103/PhysRevB.66.033109 PACS number(s): 72.15.Rn, 05.40. $-\mathrm{a}$, 05.45.Df, 71.30. $+\mathrm{h}$

A disordered electronic system in $d>2$ dimensions can be driven from the phase of extended states to that of localized states by increasing the strength of disorder. This transition, bearing the name of Anderson, is characterized by remarkably rich critical properties. In particular, the eigenfunctions at the critical point show strong fluctuations and represent multifractal distributions.

These fluctuations can be quantitatively characterized by a set of inverse participation ratios (IPRs), $P_{q}$ $=\int d^{d} r|\psi(\mathbf{r})|^{2 q}$. Using the renormalization group in $d=2^{q}$ $+\epsilon$ dimensions $(\epsilon \ll 1)$, Wegner found ${ }^{1}$ that the ensembleaveraged IPRs, $\left\langle P_{q}\right\rangle$, show, at criticality, an anomalous scaling with respect to the system size $L,\left\langle P_{q}\right\rangle \propto L^{-\tilde{\tau}_{q}}$, where ${ }^{2}$

$$
\tilde{\tau}_{q}=(q-1) d-q(q-1) \epsilon+O\left(\epsilon^{4}\right) .
$$

Equation (1) is written for the case of unbroken time-reversal symmetry corresponding to the orthogonal ensemble which we consider in the paper. According to Eq. (1), the fractal dimensions $\widetilde{D}_{q}=\tilde{\tau}_{q} /(q-1)$ are different from the spatial dimension $d$ and depend on $q$, manifesting the multifractal character of the wave-function intensity $|\psi(\mathbf{r})|^{2}{ }^{3}$ It is customary to characterize such a distribution by its singularity spectrum determined by the Legendre transform of $\tilde{\tau}_{q}$, yielding ${ }^{3,4}$

$$
\tilde{f}(\alpha)=d-(d+\epsilon-\alpha)^{2} / 4 \epsilon+O\left(\epsilon^{4}\right) .
$$

In the present paper we study how the wave-function statistics and, in particular, the multifractality spectrum, evolve with increasing spatial dimensionality, when the transition shifts from the weak-disorder range (as in $d=2+\epsilon$ ) to the strong-disorder one. A numerical study of the Anderson transition in higher-dimensional systems is of special interest since no analytical results for the critical behavior in high dimensions are available. In contrast to conventional secondorder phase transitions for which the mean-field treatment becomes valid above the upper critical dimension $d_{c}$, so that the critical exponents are $d$ independent for $d \geqslant d_{c}$, the usual mean-field approach fails in this case. The model has been solved on the Bethe lattice, ${ }^{5}$ which is believed to correspond to the limit $d=\infty$. While for the critical exponent of the localization length the conventional mean-field value $\nu$ $=1 / 2$ was obtained, a very unusual non-power-like critical behavior of other quantities was found (namely, an exponential vanishing of the diffusion constant and a jump in the inverse participation ratios $\left\langle P_{q}\right\rangle$ with $q=2,3, \ldots$ at the mobility edge). It was, however, shown in Ref. 6 that these results are intimately related to the spatial structure of the Bethe lattice. On this basis, it was argued that although the symmetry-breaking description of the transition in terms of an order-parameter function ${ }^{5}$ is of general validity, the peculiar critical behavior found in Ref. 5 is an artifact of the Bethe lattice and should take a power-law form for any finite $d<\infty$. Assuming that the critical indices should match at $d$ $\rightarrow \infty$ the Bethe lattice behavior (which corresponds to $\tilde{\tau}_{q}$ $=0$ for $q \geqslant 2$ and to the value $s=\infty$ for the conductivity exponent), one then concludes that the upper critical dimension is $d_{c}=\infty$ (Ref. 6, also see Ref. 7). While available numerical results for the value of $\nu^{8,9}$ and for the form of the critical level statistics ${ }^{9}$ in four dimensions are consistent with this conjecture, its rigorous justification and a systematic analytical study of the transition for large $d$ are still missing.

To investigate the dimensionality dependence of the critical statistics of wave functions, we have performed numerical simulations of three-dimensional (3D) and 4D tightbinding models with periodic boundary conditions and a box distribution of site energies. We have taken values $W_{c}$ $=16.5$ for three dimensions ${ }^{10}$ and $W_{c}=35$ for four dimensions $^{8,9,11}$ for the critical disorder. We have calculated wave functions with energies close to zero by diagonalizing the Hamiltonian using efficient numerical packages. ${ }^{12,13}$ Thereby we could average over an ensemble that contained typically $10^{3}$ samples. From every sample 128 wave functions with energy close to zero have been taken into account.

We begin by showing in Fig. 1 the evolution of the distribution $\mathcal{P}\left(\ln P_{2}\right)$ in three dimensions with the linear size $L$ of the system from $L=8$ to 80 . It is clearly seen that at large $L$ the distribution acquires a scale-independent limiting form and simply shifts along the $x$ axis without changing its shape. The scale-invariance of the IPR distribution at criticality was conjectured in Ref. 14. This conjecture was questioned in Ref. 15, where numerical simulations of the IPR distribution in three dimensions were performed, with the conclusion that the fractal dimension $D_{2}$ is not a well-defined quantity but rather shows strong fluctuations with a root-mean-square deviation $\operatorname{rms}\left(D_{2}\right) \sim 1$. In terms of the IPR distribution the 


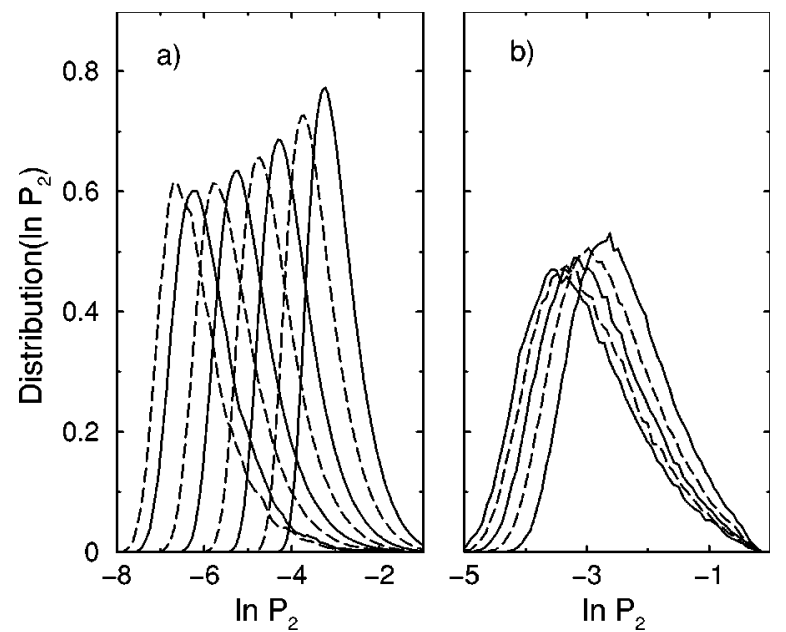

FIG. 1. IPR distribution in (a) three dimensions (system sizes $L=8,11,16,22,32,44,64$, and 80 ) and (b) four dimensions ( $L$ $=8,10,12,14$, and 16).

statement of Ref. 15 would mean that the rms deviation $\sigma_{2}$ $\equiv\left[\operatorname{var}\left(\ln P_{2}\right)\right]^{1 / 2}$ scales in the same way as $\left\langle-\ln P_{2}\right\rangle \propto \ln L$ in the limit of large $L$. However, a detailed analytical and numerical study of the wave-function statistics in a family of critical power-law random banded matrix (PRBM) ensembles has shown ${ }^{16}$ that the IPR distribution $\mathcal{P}\left(\ln P_{q}\right)$ is scale invariant, corroborating the conjecture of Ref. 14. It was argued that this is a general feature of the Anderson transition, and the conclusion of Ref. 15 was criticized as based on a not sufficiently careful numerical analysis of data obtained for too small systems. This expectation was supported by a very recent numerical study of the 3D Anderson transition, ${ }^{17}$ where a trend towards saturation of the width of the distribution $\mathcal{P}\left(\ln P_{2}\right)$ with increasing length $L$ was found. Our data [Fig. 1(a)] demonstrate the scale invariance of the limiting distribution (implying a left shift without change in form of the entire curve with increasing $L$ ) even more convincingly, since we have reached considerably larger values of $L$. The same conclusion can be drawn from the inset of Fig. 2 where the saturation of the rms deviation $\sigma_{q}(L)$ of the distribution $\mathcal{P}\left(\ln P_{q}\right)$ at $L \rightarrow \infty$ is demonstrated. Qualitatively similar results are obtained for the IPR distribution in four dimensions [Fig. 1(b)]. Though a tendency toward the saturation with increasing $L$ is clear in this case as well, the full saturation has not been reached, in view of smaller linear sizes of the system as compared to three dimensions.

To visualize the strength of the IPR fluctuations, in the main panel of Fig. 2 we show the values of the rms deviations $\sigma_{q}$, which characterize the width of the distribution functions $\mathcal{P}\left(\ln P_{q}\right)$, extrapolated to $L \rightarrow \infty$. In $d=2+\epsilon$ dimensions $\sigma_{q}$ can be calculated analytically following, ${ }^{14}$ with the result

$$
\sigma_{q}^{2}=8 \pi^{2} a_{2} \epsilon^{2} q^{2}(q-1)^{2}, \quad q \ll q_{c}=(2 / \epsilon)^{1 / 2},
$$

where $a_{2}=0.00387$ for the periodic boundary conditions. Here $q_{c}$ is the value of $q$ corresponding to the root $\alpha_{-}$of the

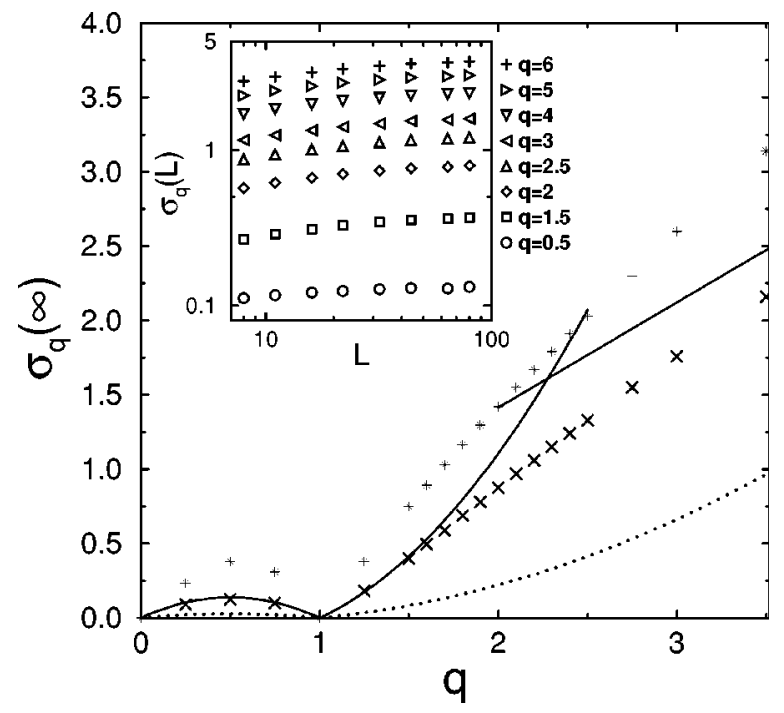

FIG. 2. The rms deviation $\sigma_{q}$ of $\ln P_{q}$ extrapolated to $L \rightarrow \infty$ in three dimensions $(X)$ and four dimensions (stars). The dotted line is the analytical result [Eq. (3)] for $\epsilon=0.2$; the full lines represent Eqs. (3) and (5) with $\epsilon=1$. Inset: evolution of $\sigma_{q}$ with $L$ in three dimensions for selected values of $q=0.5,1.5,2,2.5,3,4,5,6$. The leading finite-size correction of all data has the form $L^{-y}$ with $y$ $=0.25-0.5$ for three dimensions and $y=0.1-0.4$ in four dimensions. The numerical error in the extrapolated values of $\sigma_{q}(\infty)$ is as large as $10 \%$ due to the uncertainty in $y$.

singularity spectrum $\tilde{f}(\alpha)$, i.e., $q_{c}=\tilde{f}^{\prime}\left(\alpha_{-}\right)$and $\tilde{f}\left(\alpha_{-}\right)=0$. For $q \gg q_{c}$ the IPR distribution is dominated by its slowly decaying power-law "tail,"

$$
\mathcal{P}\left(P_{q} / P_{q}^{\mathrm{typ}}\right) \propto\left(P_{q} / P_{q}^{\mathrm{typ}}\right)^{-1-x_{q}}, \quad P_{q} \gtrsim P_{q}^{\mathrm{typ}},
$$

where $x_{q}=q_{c} / q .{ }^{16}$ This yields $\sigma_{q}=q / q_{c}$, or in $2+\epsilon$ dimensions,

$$
\sigma_{q}=(\epsilon / 2)^{1 / 2} q, \quad q \gg(2 / \epsilon)^{1 / 2} .
$$

In addition to the numerical results for three and four dimensions, in Fig. 2 we present the result [Eq. (3)] of the $\epsilon$ expansion for $d=2.2$. Furthermore, we show the small and large- $q$ analytical asymptotics [Eqs. (3) and (5)] with $\epsilon=1$. Of course, the $\epsilon$ expansion is only justified parametrically for $\epsilon \ll 1$. Nevertheless, we see that it still describes very reasonably the IPR fluctuations at the Anderson transition in three dimensions.

Having demonstrated that the distributions of the IPRs, $P_{q}$, are scale invariant at criticality, so that the fractal dimensions are well defined, we are prepared to analyze the form of the multifractal spectra. We use the numerical procedure described in Ref. 18, where it was applied to the quantum Hall plateau transition. Specifically, we calculate the ensemble-averaged IPR $\left\langle P_{q}\right\rangle$ for different system sizes $L$, extract $\tilde{\tau}_{q}$ and $\tilde{f}(\alpha)$, and extrapolate to the thermodynamic limit $L \rightarrow \infty$ in order to eliminate the finite-size corrections. We refer the reader to Ref. 18 for more details of the procedure, and for a discussion of its advantages as compared to the box-counting calculations performed in earlier publications. 


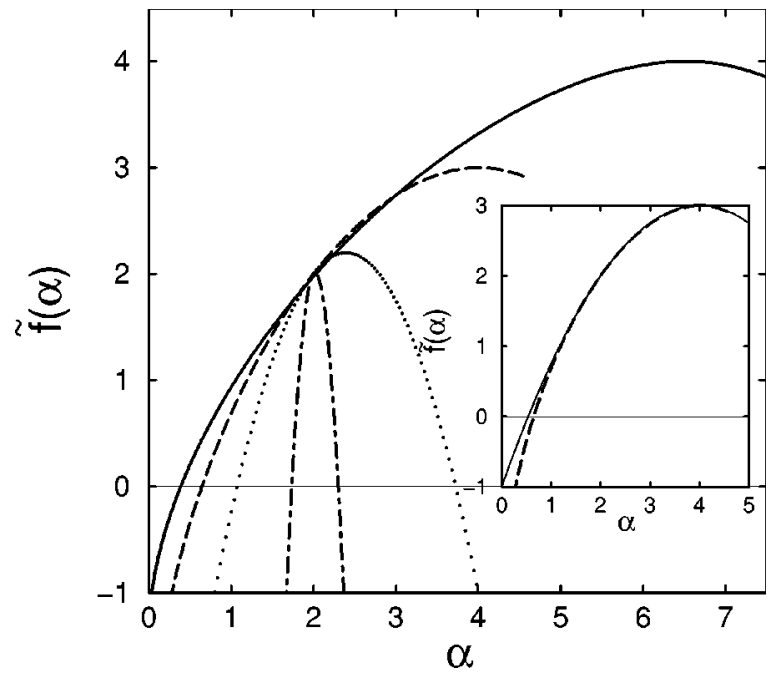

FIG. 3. Singularity spectrum $\tilde{f}(\alpha)$ in three dimensions (dashed) and four dimensions (full line). To illustrate the evolution of the spectrum from $d=2$ to 4 , analytical results for $d=2+\epsilon$ are shown for $\epsilon=0.2$ (dotted line) and $\epsilon=0.01$ (dot-dashed line). Inset: comparison between $\tilde{f}(\alpha)$ for three dimensions and Eq. (2) with $\epsilon=1$ (solid line).

The obtained results for $\tilde{f}(\alpha)$ in three and four dimensions are shown in Fig. 3. To illustrate the evolution of the spectrum in the whole range from $d=2$ to 4 , the analytical results [Eq. (2)] for $2+\epsilon$ dimensions with $\epsilon=0.2$ and 0.01 are also shown. In Fig. 4 the corresponding results for the fractal dimensions $\widetilde{D}(q)$ are presented. We see that with increasing dimensionality the singularity spectrum $\tilde{f}(\alpha)$ broadens. This is not surprising: with increasing $d$ the transition moves further in the region of strong disorder, implying stronger multifractality. What is much less obvious is that in the range $\alpha \leq 2$ the $\tilde{f}(\alpha)$ curve shifts to the left with increasing $d$. This corresponds to the fact that the fractal exponents

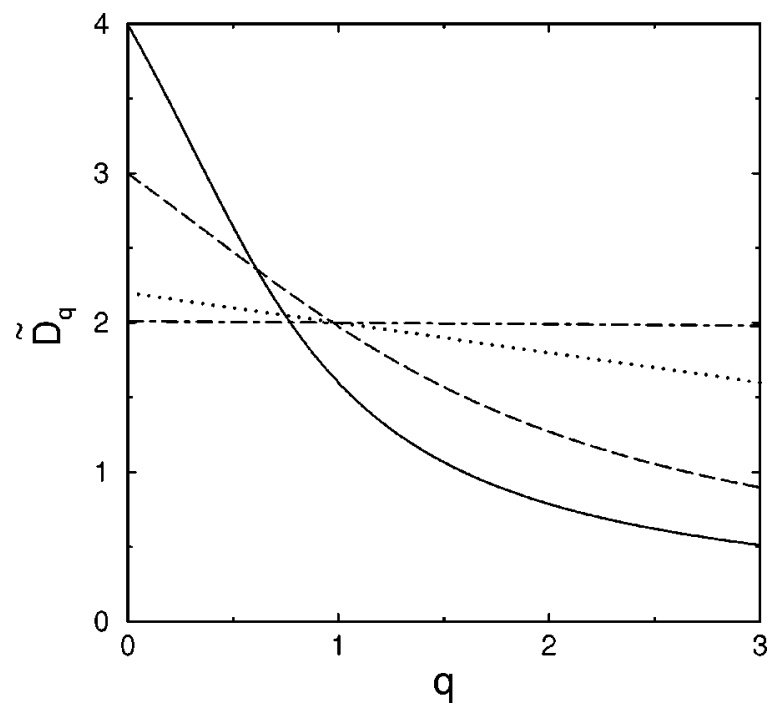

FIG. 4. Fractal dimensions $\widetilde{D}_{q}$ in three dimensions (dashed line) and four dimensions (full line). Analytical results for $d=2+\epsilon$ with $\epsilon=0.2$ (dotted line) and $\epsilon=0.01$ (dot-dashed line) are also shown.
$\widetilde{D}_{q}$ with $q \geq 1$ decrease with increasing $d$. In particular, for the exponent $\widetilde{D}_{2}$ determining the spatial dispersion of the diffusion coefficient at criticality we find $\widetilde{D}_{2}=1.3 \pm 0.05$ in three dimensions and $\widetilde{D}_{2}=0.9 \pm 0.15$ in four dimensions. ${ }^{19}$ It is worth mentioning that the $\epsilon$ expansion [Eq. (2)] with $\epsilon=1$ describes the 3D spectrum remarkably well (though with detectable deviations; see the inset of Fig. 3). In particular, the position of the maximum, $\alpha_{0}=4.03 \pm 0.05$, is very close to its value $\alpha_{0}=d+\epsilon$ implied by Eq. (2). As expected, in four dimensions the deviations from the parabolic shape are much more pronounced and $\alpha_{0}=6.5 \pm 0.2$ differs noticeably from 6 .

The obtained value of $\widetilde{D}_{2}=1.3 \pm 0.05$ in three dimensions is considerably smaller than what was found in the earlier numerical studies ${ }^{20}$ where values in the range $1.4-1.8$ were reported. The reasons for this are as follows. In the earlier works the spectrum $f(\alpha)$ of individual eigenstates was studied via the box-counting procedure. While in the limit $L$ $\rightarrow \infty$ the spectrum $f(\alpha)$ defined in this way should reproduce the part of the $\tilde{f}(\alpha)$ curve lying above the $x$ axis, for a finite $L$ the finite-size effects affect $f(\alpha)$ strongly, especially in the region close to the zero $\alpha_{-}$of $\tilde{f}(\alpha)$. As a result, such a method not only fails to yield the exponents $\widetilde{D}_{q}$ with $q>q_{c}$ but also leads to very large errors in determination of $D_{q}$ with $q$ smaller than but close to $q_{c}$. In particular, in three and four dimensions we find that $q_{c}$ is close to 2, explaining large errors in earlier results for $q=2$ in three dimensions. As to the $4 \mathrm{D}$ case, we are not aware of any previous studies of the wave-function statistics at the Anderson transition.

Let us discuss now what our findings imply for the high$d$ behavior of the critical wave-function statistics. First of all, we note that our numerical observation that $\widetilde{D}_{q}$ with $q \geq 1$ decreases with increasing dimensionality confirms the above-mentioned expectation that they should tend to zero in the limit $d \rightarrow \infty$. In view of this, an analogy with the PRBM ensemble with the parameter $b \ll 1$ is very instructive. This ensemble can be considered as describing a 1D chain with random long-range hopping whose rms amplitude is $b / r$, where $r$ is the distance. The model is critical for arbitrary 0 $<b<\infty$, and can be studied analytically in both limits $b$ $\gg 1$ and $b \ll 1$. The latter case is relevant to the issue under discussion, and we remind the reader of the key results ${ }^{16}$ concerning the wave-function statistics at $b \ll 1$. (The case $b \gg 1$ is to a large extent analogous to the Anderson transition in $2+\epsilon$, dimensions with $\epsilon \ll 1$.) Specifically, the scaleinvariant critical distribution $\mathcal{P}\left(P_{q} / P_{q}^{\mathrm{typ}}\right)$ becomes $b$ independent in the limit $b \ll 1$. Furthermore, the exponents $\tilde{\tau}_{q}$ with $q>1 / 2$ are proportional to $b$ in the small- $b$ limit:

$$
\tilde{\tau}_{q}=2 b \widetilde{T}_{q}, \quad \widetilde{T}_{q}=2 \Gamma(q-1 / 2) / \pi^{1 / 2} \Gamma(q-1) .
$$

Correspondingly, the singularity spectrum $\tilde{f}(\alpha)$ acquires, for $\alpha \ll 1$, the form

$$
\tilde{f}(\alpha)=2 b \widetilde{F}(\alpha / 2 b),
$$


where $\widetilde{F}(A)$ is the Legendre transform of $\widetilde{T}(q)$, with the asymptotics $F(A) \simeq-1 / \pi A$ as $A \rightarrow 0$ and $F(A) \simeq A / 2$ as $A$ $\rightarrow \infty$. The smallness of the fractal exponents, $\tilde{\tau}_{q} \ll 1$, reflects a very sparse structure of the eigenstates formed by resonances (spikes) with a hierarchy of distances between them, $r_{1} \ll r_{2} \ll \ldots$, such that $\ln r_{i+1} / r_{i} \sim 1 / b .^{16}$

After this reminder we return to the Anderson transition in high dimensionality. The smallness of the fractal exponents, $\widetilde{D}_{q} \ll 1$ at $d \gg 1$ implies, in close similarity to the PRBM model, that the corresponding critical eigenstates have a resonance structure with a hierarchy of scales $\ln r_{i+1} / r_{i}$ $\sim 1 / b_{d}$ and with a dimensionality-dependent parameter $b_{d}$ satisfying $b_{d} \rightarrow 0$ as $d \rightarrow \infty$. (In contrast to the PRBM model, where the emergence of resonances was due to direct longrange hopping processes, now it should be determined by interference of all possible paths connecting two sites on a $d$-dimensional lattice.) The sparse resonance structure of the eigenstates allows us to use the analogy with the PRBM model and to make the following conjectures. (i) The scaleinvariant distribution $\mathcal{P}\left(P_{q} / P_{q}^{\mathrm{typ}}\right)$ for $q>1 / 2$ becomes essentially $d$ independent at $d \gg 1$. (ii) The fractal exponents at $d$ $\gg 1$ and for $q>1 / 2$ take the form $\tilde{\tau}_{q}(d)=2 b_{d} \widetilde{T}_{q}$, i.e., they depend on $d$ only through the overall factor $b_{d}$ (satisfying $b_{d} \rightarrow 0$ as $\left.d \rightarrow \infty\right)$. Correspondingly, the singularity spectrum scales at large $d$ as $\tilde{f}(\alpha)=2 b_{d} \widetilde{F}\left(\alpha / 2 b_{d}\right)$. Though we are fully aware of the speculative character of these conjectures at the present stage, we expect that they will stimulate further numerical and analytical work, which should finally resolve the question posed by the Anderson transition in high $d$.

In conclusion, we have studied the statistics of critical wave functions at the Anderson transition in three and four dimensions. The distribution of the inverse participation ratio $P_{q}$ was demonstrated to acquire a scale-invariant form in the limit of large system size. As a convenient measure of the strength of the IPR fluctuations, we have evaluated the rms deviation $\sigma_{q}$ of $\ln P_{q}$ and found a result matching well analytical predictions for $2+\epsilon$ dimensions with $\epsilon \ll 1$. Calculating the ensemble-averaged IPR values, $\left\langle P_{q}\right\rangle$, we have determined the spectrum of fractal dimensions $\tilde{\tau}_{q} \equiv \widetilde{D}_{q}(q-1)$ and the singularity spectrum $\tilde{f}(\alpha)$ characterizing the multifractal properties of the wave functions. In particular, we have found $\widetilde{D}_{2}=1.3 \pm 0.05$ in three dimensions and $\widetilde{D}_{2}=0.9$ \pm 0.15 in four dimensions. More generally, our results indicate that the dimensions $\widetilde{D}_{q}$ with $q \gtrsim 1$ decrease with increasing spatial dimensionality $d$. On this basis, we have formulated two conjectures concerning the wave function statistics at criticality in the large- $d$ limit.

It is a pleasure to acknowledge help with the implementation and usage of the Watson Sparse Matrix Package by A. Gupta and M. Krauss. This work was supported by the SFB195 and the Schwerpunktprogramm "Quanten-HallSysteme" der Deutschen Forschungsgemeinschaft.
*Also at Petersburg Nuclear Physics Institute, 188350 St. Petersburg, Russia.

${ }^{1}$ F. Wegner, Z. Phys. B: Condens. Matter 36, 209 (1980).

${ }^{2}$ Following the notations introduced in Ref. 16, we indicate by tilde that the index $\tilde{\tau}_{q}$ in Eq. (1) corresponds to the ensembleaveraged IPR rather than to the typical one, $P_{q}^{\text {typ }}$. For a detailed discussion of the necessity to distinguish between $\left\langle P_{q}\right\rangle$ and $P_{q}^{\text {typ }}$ the reader is referred to Ref. 16 .

${ }^{3}$ C. Castellani and L. Peliti, J. Phys. A 19, L429 (1986).

${ }^{4}$ F. Wegner, Nucl. Phys. B 280, 210 (1987).

${ }^{5}$ M.R. Zirnbauer, Phys. Rev. B 34, 6394 (1986); Nucl. Phys. B 265, 375 (1986); K.B. Efetov, Zh. Éksp. Teor. Fiz. 92, 638 (1987) [Sov. Phys. JETP 65, 360 (1987)]; Physica A 167, 119 (1990); A.D. Mirlin and Y.V. Fyodorov, Nucl. Phys. B 366, 507 (1991).

${ }^{6}$ A.D. Mirlin and Y.V. Fyodorov, Phys. Rev. Lett. 72, 526 (1994); J. Phys. I 4, 655 (1994).

${ }^{7}$ C. Castellani, C. Di Castro, and L. Peliti, J. Phys. A 19, L1099 (1986).

${ }^{8}$ M. Schreiber and H. Grussbach, Phys. Rev. Lett. 76, 1687 (1996).

${ }^{9}$ I.K. Zharekeshev and B. Kramer, Ann. Phys. (Leipzig) 7, 442 (1998).

${ }^{10}$ B. Kramer and A. MacKinnon, Rep. Prog. Phys. 56, 1496 (1993); K. Slevin, T. Ohtsuki, and T. Kawarabayashi, Phys. Rev. Lett. 84, 3915 (2000), and references therein.

${ }^{11}$ P. Markoš and M. Henneke, J. Phys.: Condens. Matter 6, L765 (1994).
${ }^{12}$ R.B. Lehoucq, D. Sorensen, and C. Yang, ARPACK Users Guide (SIAM, Philadelphia, 1998).

${ }^{13}$ A. Gupta, M. Joshi, and V. Kumar, IBM Report No. RC 22038 (98932), http://www.cs.umn.edu/ agupta/doc/wssmp-paper.ps (2001).

${ }^{14}$ Y.V. Fyodorov and A.D. Mirlin, Phys. Rev. B 51, 13403 (1995); A.D. Mirlin, Phys. Rep. 326, 259 (2000).

${ }^{15}$ D.A. Parshin and H.R. Schober, Phys. Rev. Lett. 83, 4590 (1999).

${ }^{16}$ F. Evers and A.D. Mirlin, Phys. Rev. Lett. 84, 3690 (2000); A.D. Mirlin and F. Evers, Phys. Rev. B 62, 7920 (2000).

${ }^{17}$ E. Cuevas, M. Ortuño, V. Gasparian, and A. Pérez-Garrido, Phys. Rev. Lett. 88, 016401 (2002).

${ }^{18}$ F. Evers, A. Mildenberger, and A.D. Mirlin, Phys. Rev. B 64, 241303(R) (2001).

${ }^{19}$ We note that our results for four dimensions show that a statement on a lower bound for the localization length exponent, $\nu$ $>2 / D_{2}$, which can be found in the literature [see, in particular, M. Janssen, Int. J. Mod. Phys. B 8, 943 (1994); M. Janßen, O. Viehweger, U. Fastenrath, and J. Hajdu, Introduction to the Theory of the Integer Quantum Hall Effect (VCH, Weinheim, 1994), Chap. 12.6] is incorrect.

${ }^{20}$ M. Schreiber and H. Grussbach, Phys. Rev. Lett. 67, 607 (1991); H. Grussbach and M. Schreiber, Phys. Rev. B 51, 663 (1995); T. Ohtsuki and T. Kawarabayashi, J. Phys. Soc. Jpn. 66, 314 (1996); T. Brandes, B. Huckestein and L. Schweitzer, Ann. Phys. (Leipzig) 5, 633 (1996); T. Terao, Phys. Rev. B 56, 975 (1997). 